\title{
Determination of Surface Heat Flow in Mazesta (USSR) (*)
}

\author{
H. A. Lubimova, L. M. Lusova, F. V. Firsov, \\ G. N. Starikova, A. P. Shushpanov (**) \\ Ricevuto il 7 dicembre 1960
}

A great number of temperature measurements in deep bore-holes are made on the territory of the Soviet Union. The results of these measurements are partly systematized in the work "Problems of Geothermie" (1). However they can be hardly used for the calculations of heat flow because of the absence of the corresponding determinations of the thermal conductivity of rock cores taken directly from these boreholes. In the present paper the temperature gradient and the thermal conductivity of samples of rocks for the same place are determined and by means of these data the value of the thermal flow is estimated.

For temperature measurements the electric resistance thermometer was used that is designed by Dergunov I. D. and improved for the conditions of work in deep bore-holes (up to $4-5 \mathrm{~km}$ ) at rather high pressures and temperatures. The electrical resistance was measured by means of the compensation methods by a special potentiometer and a high sensitive mirror galvanometer. Four bringing wires are used for doing away with the erratic currents and the influence of the wires. As a result of it the accuracy of the temperature measurements is about $0.01^{\circ} \mathrm{C}$. Fig. 1 represents the exterior of the apparatus and the thermometer. The thermometer is a hollow cylinder on the surface of which a copper wire is winded bifilarily, whose resistance is measured. When the cylinder is buried into a bore-hole it is washed by a solution from outside and inside. Together with the application of ftorplast isolation this decreases the thermal inertia of the thermometer down to 1.5 sec. For the sake of strength annular justs are made on the cylinder. The thermometer stands the pressure up to $600 \mathrm{~atm}$ and the temperature up to $200^{\circ} \mathrm{C}$.

(*) Paper read at the Helsinky Assembly of the I.U.G.G., 1960.

(**) Institute of Earth's Physics Academy of Science USSR). 
In the process of the determinations of the temperature the requirements of the bore-holes measurements' methodics were strictly observed; the measurements were made by a point method in bore-holes that were in rest for 8 to 10 months.

As a total about 20 bore-holes with the depths from 400 to $2600 \mathrm{~m}$ were investigated. Here the data for the 3 bore-holes Mazesta-Hosta

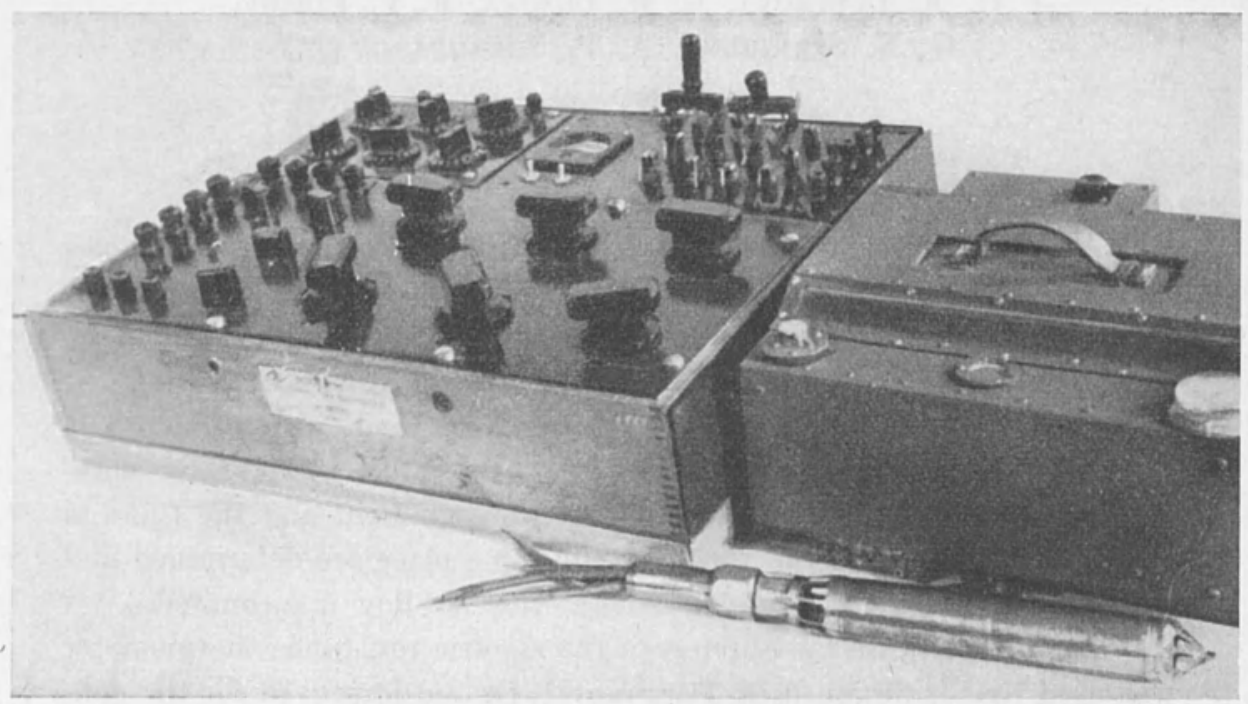

Fig. 1 - The exterior of the Apparatus and thermometer for the measurement of temperature.

(Caucasus) are given. Temperatures were measured twice in it in 1955 and 1957 and yielded the same results. As to its geology the region Mazesta refers to a great tectonic depression submerged below the level of the Black sea in its South-Western part and extended in the direction of the general striking of the Caucasus for the length of 60 to $65 \mathrm{~km}$ and amounting its maximum width of 20 to $25 \mathrm{~km}$. The complete formation of the depression and the submergence of its part below the sea level are dated to the end of the Tertiary and the beginning of the Quaternary ( $\left.{ }^{2}\right)$. Fig. 4, represents the lithological and stratigraphical cross-section of the bore-hole $3 \mathrm{~T}$.

The thermal constants of rock cores were determined by the impulse sound method. In a core sample two holes are drilled at a distance $r$ from each other. One is for an electric heater and the other for ther- 
mopair. The exterior of the apparatus is represented in fig. 2.4 current impulse is transmitted through the heater during 3 to 4 sec which creates a practically instantaneous source of heat since the time of the thermal impulse propagation from the source to the observations point is of the order of a hundred seconds. The movement of the light spot of a mirror

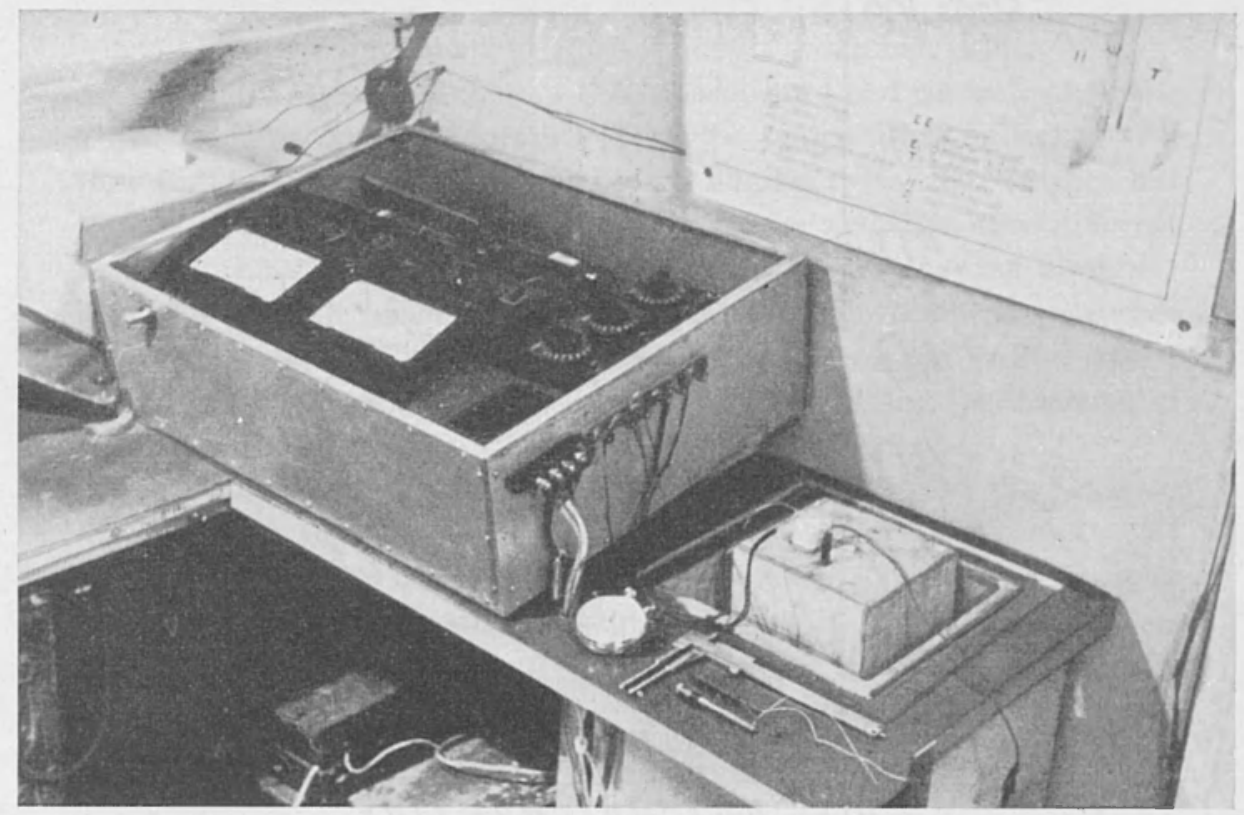

Fig. 2 - Photograph of the apparatus for the measurement of the thermal constants of rocks

galvanometer indicates the moment when the maximum temperature is reached at a given point. The time interval $t_{\max }$ and the value of the maximum temperature $\theta_{\max }$ at a given point of observation are determined and used for the determination of the thermal diffusivity $k$

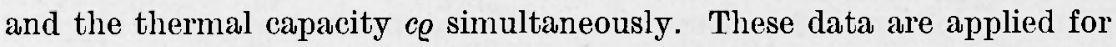
the calculation of the thermal conductivity $\lambda=k c \varrho$. This method las essential advantages over the stationary method, the so-called "divided bar" method. It does not require a through polishing of a core sample before the experiment, which greatly disturbs its natural state. The preparation of the sample for the experiments consists only in boring of holes for the heater and the thermopair. The experiment takes some 
minutes. A pressing spring being a heater's spiral at the same tirne provides a close contact with the rock. This method permits to determine the heat conductivity both along and across the rocks striking and can be used for the determination of the thermal conductivity "in situ".

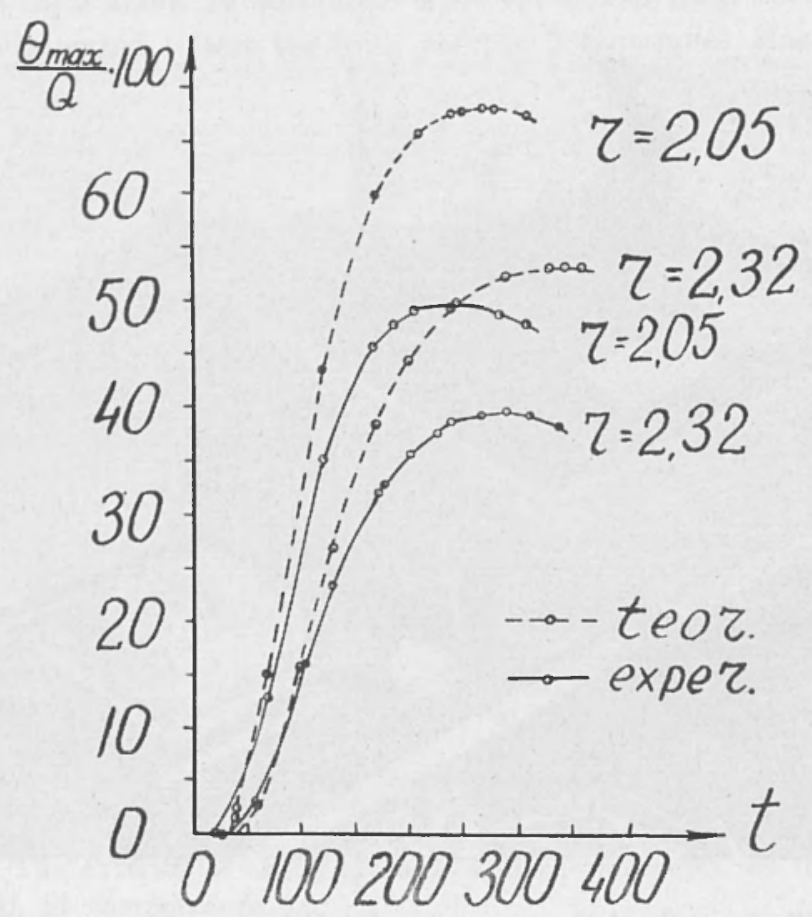

Fig. 3 - Temperature variation with time at the different distances $r$ from the source.

The heater is a kind of a rod with the radius of $0,3 \mathrm{~cm}$ and the length $2 l=5 \mathrm{~cm}$. For calculation such a source can be considered neither point nor spherical. The formula for the temperature distribution about an infinitely long and thin source in an infinite medium was used

$$
\theta(r, t)=\frac{Q}{4 \pi c \varrho k t} \exp \left(-\frac{r^{2}}{4 k t}\right)
$$

where $r$ is the distance between the source and the thermopair, $Q$ is the generated heat for the unit length of the source. 
The values $k$ and $c \varrho$ are determined from the conditions of the extremum $\frac{\partial \theta}{o t}=0$ by the formulas

$$
k=\frac{r^{2}}{4 t_{\max }} \quad ; \quad c \varrho=\frac{Q \cdot e^{-} 1}{\theta_{\max } \pi r^{2}}
$$

where $t_{\max }$ is the time moment when $\theta-\theta_{\max }$ at a given point.

However the comparison of the experimental and theoretical curves $\theta$ calculated by means of formula [1] of the temperature variation with time (fig. 3) shows that these curves are similar as to their pattern but they are somewhat shifted relative to the time axis and have different values of the temperature maximum. This difference does not disappear if to allow for the ultimate duration of the impulse $\Delta t$ and the ultimate size of the sample. These effects account for the error not greater than 1 per cent if the conditions $\Delta t<10$ sec are observed and the diameter of the sample at $r<3 \mathrm{sm}$.

A more essential error is due to the assumption that the heater is an infinite straight line.

The integration of the function describing the temperature influence of the instantaneous point heat source along with the cylinder surfaces with the radius $a$ and length $2 l$ yields a formula

$$
\theta(r, t)=\frac{Q}{4 \pi c \varrho k t} \exp \left(-\frac{a^{2}+r^{2}}{4 k t}\right) \cdot I_{o}\left(\frac{a r}{2 k t}\right) \cdot \operatorname{erf}\left(\frac{l}{21 k t}\right)
$$

which gives the temperature distribution about a cylindrical instantaneous source of finite dimensions, where $I_{o}(x)$ is the Bessel function of the zero order from an imaginary argument, erf is the integral of the errors. Hence the thermal diffusivity $k$ is determined graphically from the equation for $\frac{\partial \theta}{\partial t}=0$ :

$$
\frac{r^{2}+a^{2}}{4 k t_{\max }}-\frac{a r}{2 k t_{\max }} \cdot \frac{I_{1}\left(\frac{a r}{2 k t_{\max }}\right)}{I_{0}\left(\frac{a r}{2 k t_{\max }}\right)}-1+\frac{7}{21 \overline{\pi k t}} \cdot \frac{\exp \left(-\frac{l^{2}}{4 k t}\right)}{\left.\operatorname{erf} \frac{l}{\left(21^{\prime} k t\right.}\right)}
$$

using the obtained $k$ we calculate heat capacity

$$
c \varrho=\frac{Q}{4 \pi k t \theta_{\max }} \exp \left(-\frac{a+r^{4}}{4 k t_{\max }}\right) I_{o}\left(\frac{a r}{2 k t_{\max }}\right) \operatorname{erf}\left(\frac{l}{21^{\prime} k t}\right)
$$


The error due to some loss of heat through the top surface and to non-absolute contacts of the heater and the thermopair with the rock can be allowed empirically. Let us consider the simplest case and calculate the temperature curve using formula [1]. The differences of the experimental and theoretical curves of fig. 2 can be used for the determination of the correction coefficients which are inserted in formula [1]

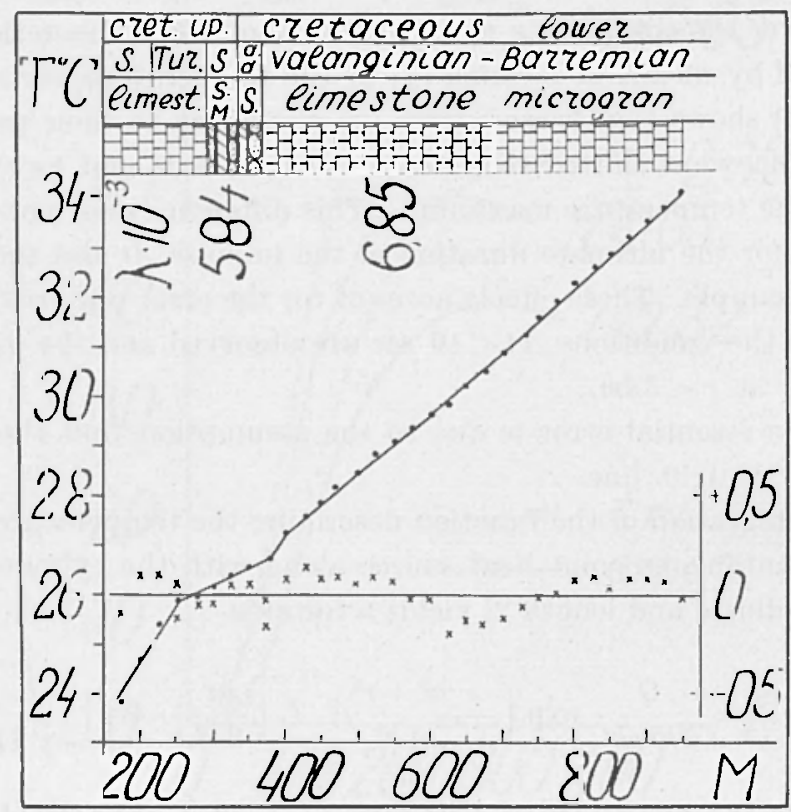

Fig. 4 - Lithological and stratigraphical cross-section of the bore-hole 3T Old Mazesta.

so that these curves coincide within the given accuracy. For example the following empirical formulas can be taken

$$
\frac{\theta}{Q}=\frac{A \exp \left(-\frac{R^{2}}{4 k t}\right)}{4 \pi c o k t} \quad ; \quad l=\frac{R^{2}}{4 t_{\max }} \quad ; \quad c \varrho=\frac{A e^{-1} \cdot Q}{\theta_{\max } \pi R^{2}}
$$

where $A(r), R(r)$ are obtained from experience for the given conditions of an experiment and allow for all the possible deviations of the idealized scheme simultaneously. The comparison of the results obtained by two methods from formulas 4-5 and 6 give discrepancies not greater than 
$\sim 5$ per cent, which is a good selfcheck. An independent study of one and the same specimen of chalk by our method and by the method of regular regime in another Institute gave a rather satisfactory coincidence of the results.

Temperature measurements in the bore-hole $3 \mathrm{~T}$ old Mazesta were made to the depth of $2165 \mathrm{~m}$ and with the interval of $25 \mathrm{~m}$ The results are given in table 1 . The core samples were selected in the near lying bore-hole $4 \mathrm{~T}$ located at the distance of $\sim 200 \mathrm{~m}$. Both lithologically and stratigraphycally the selected core-samples correspond to rocks in the bore-hole $3 \mathrm{~T}$ in the range of 175 to $950 \mathrm{~m}$.

Three intervals with different temperature gradients answering the alternation of layers can be distinguished on the temperature curves (fig. 4) corresponding to these depths. The valued grad $T$ and $T_{o}$ in the formula

$$
T=T_{o}+D \cdot \operatorname{grad} T
$$

is determined by the least square method for each interval, where $D$ is the depth, $T$ is the temperature at a given depth, $T_{o}$ is the constant designating the value of temperature extrapolated to the level of the Earth's surface.

Thus the temperature curve can be represented by the following equations:

a) in the range of 175 to $250 \mathrm{~m}$

$$
T=19,01+2,812 \cdot 10^{-1} D, \operatorname{grad} T=2,812 \cdot 10^{-40} \mathrm{C} / \mathrm{cm}=28,12^{\circ} \mathrm{C} / \mathrm{km}
$$

b) in the range of 250 to 375

$$
\begin{aligned}
& T=24,23+0,658 \cdot 10^{-4} \mathrm{D}, \operatorname{grad} T=0,658 \cdot 10^{-40} \mathrm{C} / \mathrm{cm}=6,58^{\circ} \mathrm{C} / \mathrm{km} \\
& \quad c) \text { in the range of } 375 \text { to } 950
\end{aligned}
$$

$$
T=22,10+1,264 \cdot 10^{-4} \mathrm{D}, \operatorname{grad} T=1,264 \cdot 10^{-40} \mathrm{C} / \mathrm{cm}=12,64^{\circ} \mathrm{C} / \mathrm{km}
$$

The deviations of $\Delta T$ calculated from these formulas of the temperature values from the observed ones are represented by crosses in fig. 4 .

An increase of the gradient in the upper range $a$ ) may be connected with the influence of the relief and the other fators. Therefore we shal not take into account this range of depths and calculate the thermal flow for the range of 250 to $950 \mathrm{~m}$. 
The determination of the average gradient for the II and III intervals by the least suquare method gives for the range of 250 to $950 \mathrm{~m}$ $T=22,40+1,224 \cdot 10^{-4} D$,

$$
\operatorname{grad} T=12,24^{\circ} \mathrm{C} / \mathrm{km} \text {. }
$$

The deviation of $\Delta T_{2}$ observed temperature from the calculated using formulas [8] is given in table 4, column 1. The standard error is $0.11^{\circ} \mathrm{C}$.

Below the values of the heat conductivity of rock cores across the striking are represented.

$250-290$

$$
\begin{aligned}
& 0,00725 \\
& 0,00653 \\
& 0,00689 \text { Limestone of the Turon }
\end{aligned}
$$

$290-315$

$$
\text { среднее } \frac{0,00517}{0,00594} \text { Marls of the senoman tufaceous sandstone }
$$

$315-328$

0,00417

$328-375$

0,00664

0,00627

среднее 0,00646 Marls of the Alb and the Apt

$375-950$

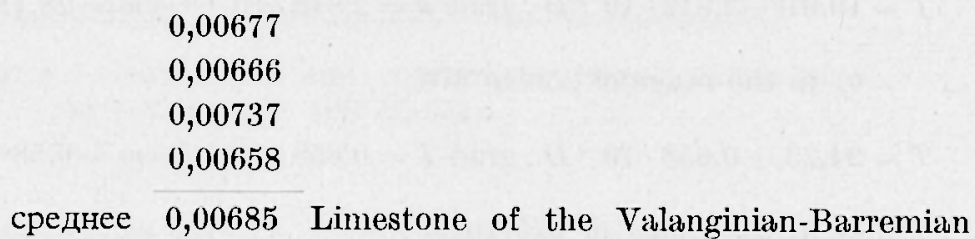

The average value of the thermal conductivity in the range of 250 to $950 \mathrm{~m}$ consisting of a great number of layers was calculated by means of the formula

$$
\begin{aligned}
& \lambda=\frac{D_{i}-D_{k}}{\frac{D_{i}}{\lambda_{i}} \div \frac{D_{i}+1}{\lambda_{i}+1} \div \ldots \frac{D_{k}}{\lambda_{k}}}=\frac{4000}{\frac{4000}{6,89 \cdot 10^{-3}} \div \frac{57500}{6,85 \cdot 10^{-3}}}= \\
& =6,64 \cdot 10^{-3} \frac{\mathrm{cal}}{\mathrm{cm}^{20} \mathrm{C} \mathrm{sec}} \pm 0,60 \cdot 10^{-3} \mathrm{~cm}^{20} \mathrm{C} \mathrm{sec} .
\end{aligned}
$$


The average values of $\lambda$ for the II and III intervals are equal to $5,84 \cdot 10^{-3}$ and $6,85 \cdot 10^{-3} \frac{\mathrm{cal}}{\mathrm{cm}^{20} \mathrm{C} \mathrm{sec}}$. We obtain for the heat flow

$$
\begin{gathered}
H=\lambda \operatorname{grad} T=6,64 \\
\cdot 10^{-3} \cdot 1,22 \cdot 10^{-4}=0,81 \cdot 10^{-6} \frac{\mathrm{cal}}{\mathrm{cm}^{2} \mathrm{sec}} \pm \\
\pm 0,07 \cdot 10^{-6} \frac{\mathrm{cal}}{\mathrm{cm}^{2} \mathrm{sec}}
\end{gathered}
$$

\begin{tabular}{|c|c|c|c|c|c|}
\hline $\begin{array}{c}\text { Depth } \\
\text { m. }\end{array}$ & $\begin{array}{l}T \\
\quad \circ \mathrm{C}\end{array}$ & $\begin{array}{c}\Delta T_{1} \\
\mathrm{o}^{\mathrm{C}}\end{array}$ & $\begin{array}{c}\Delta T_{2} \\
{ }^{\circ} \mathrm{C}\end{array}$ & $\begin{array}{l}\sum \frac{D}{1} \cdot 10^{5} \\
\frac{\mathrm{cm}^{2} \text { sec }{ }^{\circ} \mathrm{C}}{\mathrm{cal}}\end{array}$ & $\begin{array}{c}\Delta T_{3} \\
\circ \mathrm{C}\end{array}$ \\
\hline $\begin{array}{l}175 \\
200 \\
225 \\
250 \\
275 \\
300 \\
325 \\
350 \\
375 \\
400 \\
425 \\
450 \\
475 \\
500 \\
525 \\
550 \\
575 \\
600 \\
625 \\
650 \\
675 \\
700 \\
725 \\
750 \\
775 \\
800 \\
825 \\
850 \\
875 \\
900 \\
925 \\
950\end{array}$ & $\begin{array}{l}23,82 \\
24,72 \\
25,45 \\
25,92 \\
26,00 \\
26,16 \\
26,41 \\
26,37 \\
26,38 \\
27,24 \\
27,61 \\
27,87 \\
28,20 \\
28,48 \\
28,82 \\
29,12 \\
29,35 \\
29,65 \\
29,80 \\
30,19 \\
30,49 \\
30,84 \\
31,20 \\
31,57 \\
31,90 \\
32,28 \\
32,61 \\
32,87 \\
33,23 \\
33,55 \\
33,85 \\
34,10\end{array}$ & $\begin{array}{c}-0,11 \\
+0,09 \\
+0,11 \\
-12+0,05 \\
-0,04 \\
-0,04 \\
+0,04 \\
+0,04 \\
-0,02-0,16 \\
+0,08 \\
+0,14 \\
+0,08 \\
+0,10 \\
+0,06 \\
+0,09 \\
+0,07 \\
-0,02 \\
-0,03 \\
-0,20 \\
-0,13 \\
-0,14 \\
-0,11 \\
-0,06 \\
-0,01 \\
0 \\
+0,07 \\
+0,08 \\
+0,03 \\
+0,07 \\
+0,07 \\
+0,06 \\
-0,01\end{array}$ & 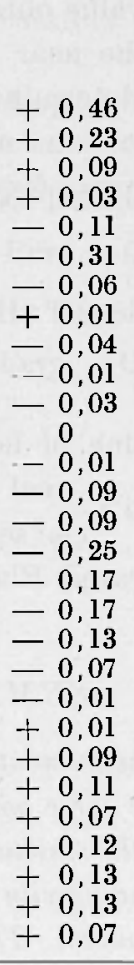 & $\begin{array}{r}42,808 \\
47,089 \\
51,370 \\
55,651 \\
59,931 \\
64,212 \\
67,863 \\
71,512 \\
75,162 \\
78,812 \\
82,461 \\
86,111 \\
89,760 \\
93,410 \\
97,060 \\
100,709 \\
104,359 \\
108,008 \\
111,658 \\
115,308 \\
118,957 \\
122,607 \\
126,256 \\
129,905 \\
133,556 \\
137,205 \\
140,855 \\
144,504 \\
148,154\end{array}$ & $\begin{array}{l}+0,62 \\
+0,35 \\
+0,16 \\
+0,05 \\
-0,14 \\
-0,38 \\
-0,12 \\
-0,05 \\
-0,05 \\
-0,06 \\
-0,08 \\
-0,04 \\
-0,05 \\
-0,12 \\
-0,12 \\
-0,27 \\
-0,18 \\
-0,18 \\
-0,13 \\
-0,07 \\
0 \\
+0,03 \\
+0,11 \\
+0,14 \\
+0,10 \\
+0,15 \\
+0,17 \\
+0,17 \\
+\quad 0,12 \\
\end{array}$ \\
\hline & & & $\pm 0,11$ & & $\pm 0,18$ \\
\hline
\end{tabular}

Table No. 1 
In accordance with a more precise method of the determination of the average heat flow through a multi-layered medium [4] we have

$$
H=\frac{T-T}{\sum_{i=1}^{n} D_{i} / \lambda_{i}}
$$

Values $\frac{\sum_{i=1}^{n} D_{i}}{\lambda_{i}}$ for each range of depths are given in table 1 , column 5. The corresponding deviations $\Delta T_{3}$ of the observed value $T$ from the calculated one using formula [9] are given in the last column.

The equation for $T$ is

$$
\begin{aligned}
& T=21,77+0,82 \cdot 10^{-6} \sum_{i=1}^{n} \frac{D}{\hat{n}_{i}} \\
& H=0,82 \cdot 10^{-6} \frac{\mathrm{cal}}{\mathrm{cm}^{2} \mathrm{sec}} \pm 0,05 \cdot 10^{-6} \frac{\mathrm{cal}}{\mathrm{cm}^{2} \mathrm{sec}}
\end{aligned}
$$

which is very close to the value obtained above. We obtained the values of the temperatures for the near located bore-holes $2 \mathrm{~T}$ New Mazesta and $2 \mathrm{~T}$ Hosta also and determined the heat flow on the base of the known lithological cross-sections and data on $\lambda$ :

1) for the bore hole $2 \mathrm{~T}$ New Mazesta $T=28,58+0,987 \cdot 10^{-4} \mathrm{D}, \operatorname{grad} T=0,987 \cdot 10^{-4} ; \mathrm{H}=0.68 \cdot 10^{-8}$

2) for the bore hole $2 \mathrm{~T}$ Hosta

$$
T=16,95+1,810 \cdot 10^{-4} \mathrm{D}, \operatorname{grad} T=1,81 \cdot 10^{-4}, \mathrm{H}=1,14 \cdot 10^{-6} \text {. }
$$

Then the average value of heat flow for Mazesta-Hosta region is equal $(0,88 \pm 0,16) \cdot 10^{-8} \frac{\mathrm{cal}}{\mathrm{cm}^{2} \mathrm{sec}}$. It is possible there is the cooling effect of the near located Black Sea and of the Mazesta's springs.

\section{$S U M M A R Y$}

The results of the measurements of the temperature and the thermal conductivity of rocks for the three boreholes Mazesta's region are described. The spesial thermometer of the resistance was used. The method of the measurements of the thermal conductivity and capacity based upon the principle of the instant linear heat source. Then calculated magnitude of heat flow is equal $0.88 \cdot 10^{-6} \mathrm{cal} / \mathrm{cm}^{2} / \mathrm{sec}$. It is likely that there is the cooling effect of the near located Black Sea and of the Mazesta's springs. 


\section{RIASSUNTO}

Sono descritti $i$ risultati delle misure della temperatura e della conduttivita termica delle rocce per $i 3$ fori pratisati nella regione della Mazesta. $E$ stato usato lo speciale termometro a resistenza. Il metodo delle misure della conduttivita e della capacita termica si basa sul principio della sorgente improvvisa lineare di calore. L'ampiezza calcolata dell'emissione di calore e uguale a $0.88 \cdot 10^{-6} \mathrm{cal} / \mathrm{cm}^{2} / \mathrm{sec}$.

\section{REFERENCES}

(1) Problems of Geothermie. Transactions of the All-Union Congress of geothermic investigations, Acad. Sci. USSR. (1959).

${ }^{2}$ ) Makarenko F. A., On the Genesis of the Sulpheretted Hydrogen Waters of Mazesta. Transactions of the Laboratory of Hydrogeological Problems Acad. Sci. USSR, v. 2, (1949).

( ${ }^{3}$ Benfield A. E., Terrestrial Heat Flow in Great Britain, Proc. Roy. Soc. $173,428-450,(1939)$. .

(') Bullard E. C., Heat Flow in South Africa. Proc. Roy. Soc. A., 173, 474-502, (1939). 